\title{
Risk categorization for oversight of laboratory-developed tests for inherited conditions
}

\author{
Kristin G. Monaghan, PhD1, Judith Benkendorf, MS², Athena M. Cherry, PhD³, Susan J. Gross, MD, \\ C. Sue Richards, $\mathrm{PhD}^{5}$, Vernon Reid Sutton, $\mathrm{MD}^{6}$ and Michael S. Watson, $\mathrm{PhD}^{2}$; a joint working \\ group of the Laboratory Quality Assurance and the Professional Practice and Guidelines \\ Committees of the American College of Medical Genetics and Genomics
}

This document represents the proposed approach of the American College of Medical Genetics and Genomics (ACMG) to classify laboratory-developed tests for inherited conditions. Risk classification has been the determinant of whether or not medical tests are overseen and regulated by the US Food and Drug Administration (FDA). Therefore, because laboratory-developed tests for germline mutations continue to proliferate without sound regulatory frameworks in place, an
ACMG-appointed workgroup of laboratorians and clinicians considered the medical risks and implications resulting from germline mutation analysis in a variety of contexts to develop the proposed approach. It is expected that the expert opinion represented in this proposed classification system will be used to guide federal agencies, policymakers, and other stakeholders.

The ACMG has categorized testing for inherited conditions by utilizing the three-tiered risk-based system (Table 1), as

Table 1 ACMGs proposed approach to risk classification and oversight of laboratory developed tests for inherited conditions

\section{Classification}

Low risk: the consequence of an incorrect result or interpretation is unlikely to lead to serious morbidity or mortality for patients or their offspring

Moderate risk: the consequence of an incorrect result or interpretation may lead to serious morbidity or mortality for patients or their blood relatives; the test methodology is well understood and independently verifiable; and interlaboratory comparisons can be performed or external proficiency testing is available.

\section{Determining factors}

The test result is typically used in conjunction with other clinical findings to establish or confirm diagnosis; no claim that the test result alone determines prognosis or direction of therapy.

The test result may be used for predicting disease progression or identifying whether a patient is eligible for a specific therapy. It includes diagnostic, presymptomatic, and predisposition genetic testing; carrier screening; preimplantation genetic diagnosis and prenatal testing, in which the confirmatory procedure may incur significant morbidity or mortality to the patient or fetus (including but not limited to invasive prenatal diagnostic procedures that may directly affect pregnancy management, outcome, and reproductive decision making).

\section{Oversight recommendations}

The laboratory internally performs analytical validation and determines adequacy of clinical validation before offering for clinical testing; the accreditor during the normally scheduled inspections will verify that the laboratory performed appropriate validation studies.

Test results require expert interpretation by an appropriately trained board-certified (ABPath/ $A B M G$ or $A B M G) M D$ or PhD. The laboratory must submit validation studies to the CMS-deemed accreditor for review, and the accreditor must make a determination that there is adequate evidence of analytical and clinical validity before the laboratory may offer the test clinically. A system needs to be developed by the American College of Medical Genetics and Genomics in conjunction with a CMS-deemed accreditor to create an algorithm for the test validation review process. The laboratory should submit validation studies demonstrating analytical and clinical validity to the CMSdeemed accreditor. Because of rapidly expanding knowledge and new techniques that improve clinical molecular testing, a rapid turnaround time for the accreditor review is necessary.

Test results require expert interpretation by an appropriately trained, board-certified (ABPath/ $A B M G$ or $A B M G) M D$ or PhD. The laboratory must submit test to the FDA for review before offering the test clinically. The CMS and accreditor determine compliance.

\begin{abstract}
High risk: the consequence of an incorrect result or interpretation could lead to serious morbidity or mortality; and the test methodology is based on a unique algorithm or proprietary method or is not independently verifiable.

The test is used to predict risk of, progression of, or patient eligibility for a specific therapy to treat a disease associated with significant morbidity or mortality; and/or the test result cannot be tied to the methods used or interlaboratory comparisons cannot be performed.
\end{abstract}


recommended by the College of American Pathologists ${ }^{1}$ and consistent with the usual FDA determination of testing-associated risk, whereby the FDA aligns risk with the medical decision made on the test results. The proposed risk categorization model of the ACMG is based on how an incorrect result might have an impact on patients and their blood relatives (including offspring). The risk model specifies determining factors for categorization and oversight recommendations for each level of risk. It should be recognized that genetic testing is a process including not only the analytical phase addressed in this document, but also preanalytical and postanalytical components, which are beyond the scope of this document. Patient harms can occur in the preanalytical phase (e.g., lack of education/counseling, disregard for the informed consent process, wrong test ordered) as well as postanalytically in the delivery of results and subsequent clinical follow-up.

Although the ACMG is in agreement with the features that the College of American Pathologists recommends to be included in the oversight framework for laboratory-developed tests, we recommend additional considerations for germline genetic testing. We recommend that all clinical molecular genetic tests fall into either the moderate-risk or high-risk category. Tests that (i) do not utilize proprietary methods or algorithms, (ii) are amenable to interlaboratory comparisons, and (iii) are evaluated by external proficiency testing should be categorized as moderate risk.
Due to the potentially serious implications of an incorrect result or interpretation for the patient and the patient's blood relatives, we recommend that all clinical molecular genetic test results be reviewed and interpreted by an individual certified in either Clinical Molecular Genetics (American Board of Medical Genetics, ABMG) or Molecular Genetic Pathology (American Board of Pathology/ABMG). The professional interpretation of test results should be provided by an individual certified in clinical genetics (ABMG), clinical cytogenetics (ABMG), clinical molecular genetics (ABMG), or molecular genetic pathology (American Board of Pathology/ABMG). In addition, we recommend that an ABMG-certified clinical geneticist and/or American Board of Genetic Counseling/ABMG-certified genetic counselor provide pre- and posttest counseling to patients, as necessary.

\section{DISCLOSURE}

The authors declare no conflict of interest. However, please note that all authors (except J.B. and M.S.W.) direct clinical testing laboratories.

\section{REFERENCE}

1. College of American Pathologists. Proposed Approach to Oversight of Laboratory Developed Tests draft proposal (4/28/2010). http://www.cap.org/ apps/docs/advocacy/ldtoversight_model.pdf Accessed 25 July 2012. 